\title{
Analysis of altered microRNA expression profiles in the kidney tissues of ethylene glycol-induced hyperoxaluric rats
}

\author{
ZHUO LIU $^{1 *}$, HONGYANG JIANG ${ }^{1 *}$, JUN YANG ${ }^{1}$, TAO WANG ${ }^{1}$, \\ YUFENG DING ${ }^{2}$, JIHONG LIU ${ }^{1}$, SHAOGANG WANG ${ }^{1}$ and ZHANGQUN YE ${ }^{1}$ \\ Departments of ${ }^{1}$ Urology and ${ }^{2}$ Pharmacy, Tongji Hospital, Tongji Medical College, \\ Huazhong University of Science and Technology, Wuhan, Hubei 430030, P.R. China
}

Received October 7, 2015; Accepted August 26, 2016

DOI: $10.3892 / \mathrm{mmr} .2016 .5833$

\begin{abstract}
Calcium oxalate stones account for $>80 \%$ of urinary stones, however the mechanisms underlying their formation remains to be elucidated. Hyperoxaluria serves an important role in the pathophysiological process of stone formation. In the present study, differences in the miRNA expression profiles between experimental hyperoxaluric rats and normal rats were analyzed, in order to identify target genes and signaling pathways involved in the pathogenesis of hyperoxaluria. Ethylene glycol and ammonium chloride was fed to male hyperoxaluric rats (EXP) and normal age-matched male rats (CON). The oxalate concentration in the urine of each experimental rat was collected every $24 \mathrm{~h}$ and measured on day 14 . Three rats exhibiting the highest concentrations were selected for microarray analysis. Microarray analysis was performed to evaluate differences in the expression of microRNA (miRNA) in the kidney tissues from EXP and CON groups, and miRNAs that exhibited a $>2$-fold or a $<0.5$-fold alteration in expression between these groups were screened for differential expression patterns according to the threshold P-values. Reverse transcription-quantitative polymerase chain reaction analysis was employed to confirm the microarray results. In order to predict the potential role of miRNAs in pathophysiological processes, gene ontology (GO), pathway and target prediction analyses were conducted. A total of 28 miRNAs were observed to be differentially expressed ( $>2$-fold change) between EXP and CON groups. Among these miRNAs, 20 were upregulated and 8 were downregulated. GO and pathway analyses revealed that
\end{abstract}

Correspondence to: Dr Tao Wang or Dr Jihong Liu, Department of Urology, Tongji Hospital, Tongji Medical College, Huazhong University of Science and Technology, 1095 Jie Fang Avenue, Wuhan, Hubei 430030, P.R. China

E-mail: tjhwt@126.com

E-mail: jhliu@tjh.tjmu.edu.cn

${ }^{*}$ Contributed equally

Key words: microRNA, gene ontology analysis, pathway analysis, hyperoxaluria, calcium oxalate stones the insulin resistance and phosphatidylinositol-bisphosphonate 3-kinase/AKT serine threonine kinase signaling pathways were potentially associated with miRNA regulation in this setting. In conclusion, the results of the present study identified differentially expressed miRNAs in hyperoxaluric rats, and provided a novel perspective for the role of miRNAs in the formation of calcium oxalate stones.

\section{Introduction}

Urolithiasis is a common urological disease that has a high recurrence rate (1). The incidence of urolithiasis in China is between 1 and $10 \%$. As calcium oxalate stones are present in $80-90 \%$ of cases, there is marked interest in elucidating the mechanisms underlying the formation of these stones. MicroRNAs (miRNAs) are a type of small non-coding RNA, that functions to silence mRNA expression and the post-transcriptional regulation of gene expression through base-pairing with complementary sequences within target mRNA molecules $(2,3)$. miRNAs target mRNA sequences for cleavage and inhibit translation by binding to the 3'-untranslated region of target mRNAs (4). It is estimated that $>30 \%$ of protein-encoding gene translation is regulated by miRNAs (5). However, to the best of our knowledge, no studies have investigated an association between miRNAs and the development of hyperoxaluria (a form of urolithiasis) in vivo. The present study hypothesized that the deregulation of miRNA expression may partially elicit cytotoxic effects in the kidneys. To the best of our knowledge, this is the first study that has investigated the miRNA expression profiles underlying formation in kidney tissues in vivo. The aim of the present study was to determine the differences in the miRNA expression profiles between hyperoxaluric rats and normal rats using miRNA microarray technology and reverse transcription-quantitative polymerase chain reaction (RT-qPCR) analysis, and to examine the interaction of miRNAs and genes using gene ontology (GO) and pathway analysis. The results may provide an insight into the mechanisms underlying the pathogenesis of calcium oxalate stone formation.

\section{Materials and methods}

Animal models. A total of 20 male Sprague-Dawley rats (275-300 g) were obtained from the Animal Experimental 
Center of Tongji Medical College, Huazhong University of Science and Technology (Wuhan, China), and were acclimated to $12 \mathrm{~h}$ light/dark cycles at $23^{\circ} \mathrm{C}$ for 1 week prior to the start of experiments in a specific pathogen-free animal house with a relative humidity of $45-55 \%$. Rats were maintained on a diet consisting of standard laboratory chow (Wuhan WanQianJiaXing Bio-Technology Co., Ltd., Wuhan, China) and had free access to the food. Rats were randomly divided into two equal groups of 10; the experimental (EXP) and control (CON) groups. Rats in the EXP group had free access to drinking water containing $1 \%(\mathrm{v} / \mathrm{v})$ ethylene glycol and $0.5 \%$ ammonium chloride for a period of two weeks in order to induce hyperoxaluria. Rats in the $\mathrm{CON}$ group were provided with tap water for two weeks. At this point, urine specimens were collected from rats at 24-h intervals using a metabolism cage, and ion chromatography (883 Basic IC plus; Metrohm AG, Herisau, Switzerland) was performed to evaluate oxalate levels according to the methods described previously (6). A total of three rats exhibited relatively high concentrations of urine oxalate levels, and all rats were anesthetized by intraperitoneal injection of pentobarbital $(40 \mathrm{mg} / \mathrm{kg})$ prior to surgery. Rats were placed in a supine position, and the kidneys were removed before they were sacrificed by neck dislocation under anesthesia at the end of surgery. Kidney tissues were fixed with 4\% paraformaldehyde (cat. no. G1101; Wuhan Goodbio technology Co., Ltd., Hubei, China), paraffin-embedded and divided into $4-\mu \mathrm{m}$ sections, before they were subject to von Kossa staining by treating with $2 \%$ silver nitrate and $5 \%$ sodium thiosulfate according to the standard staining protocol. Kidney tissues were then visualized using an inverted light microscope (IX71; Olympus Corporation, Tokyo, Japan). The present study was conducted in strict accordance with the Guide for the Care and Use of Laboratory Animals of the National Institute of Health (NIH publication no. 85-23, 1985) (7). Experiments were approved by the Tongji Medical College Council on the Animal Care Committee of Huazhong University of Science and Technology (Wuhan, China). Surgeries were performed under sodium pentobarbital anesthesia, and every effort was made to minimize suffering.

RNA isolation. Kidneys were removed, washed in several volumes of RNase-free water, decapsulated, and stored overnight at $4^{\circ} \mathrm{C}$ in 5 volumes of RNAwait (Beijing Solarbio Science and Technology, Co., Ltd., Beijing, China). Samples were preserved at $-80^{\circ} \mathrm{C}$. RNA was extracted using the miRNeasy Mini kit (Qiagen, GmbH, Hilden, Germany) according to the manufacturer's instructions. RNA quality was determined using a NanoDrop ND-1000 Spectrophotometer (NanoDrop Technologies; Thermo Fisher Scientific, Inc., Waltham, MA, USA).

miRNA microarray analysis. The Affymetrix GeneChip miRNA 4.0 array (Affymetrix, Inc., Santa Clara, CA, USA), which contains 728 rat miRNAs, was employed for the purposes of this study (Genminix Informatics Co. Ltd., Shanghai, China). Total RNA $(1 \mu \mathrm{g})$ from each sample was labeled with biotin using the Genisphere FlashTag ${ }^{\mathrm{TM}}$ Biotin HSR RNA Labeling for Affymetrix GeneChip miRNA array kit (Genisphere, Inc., Hatfield, PA, USA). Labeled miRNA was hybridized to the array for $16 \mathrm{~h}$ at $48^{\circ} \mathrm{C}$ using a plate shaker at
$60 \mathrm{rpm}$. The GeneChip was scanned using the Hewlett-Packard GeneArray Scanner G3000 7G (Hewlett-Packard, Palo Alto, CA, USA). Expression data were analyzed using the Affymetrix Expression Console software (version, 1.3.1; Affymetrix, Inc.) and normalized with the robust multiarray averaging method (8). In order to compare miRNA expression levels between EXP and CON groups, the random variance model (RVM) $t$-test was applied to identify differentially expressed genes. This is due to the fact that the RVM $t$-test increases the degrees of freedom when there is a small sample size. Following significant difference and false discovery rate (FDR) analyses, the differentially expressed genes were selected according to the $\mathrm{P}$-value threshold, where $\mathrm{P}<0.05$ was considered to indicate a significant difference $(9,10)$. The results of the miRNA microarray analysis were deposited in the NCBI Gene Expression Omnibus data repository (GEO; series accession number, GSE72135).

$R T-q P C R$ analysis. The expression of differentially expressed miRNAs identified by the microarray analysis, was verified using RT-qPCR analysis. Pre-designed stem-loop miRNA RT-qPCR primer sets that detect miR-214-3p, miR-146b-5p, miR-31a-5p, miR-369-5p and miR-141-5p expression levels, were purchased from Invitrogen (Thermo Fisher Scientific, Inc.; Table I). Total RNA was reverse transcribed using the RevertAid First Strand cDNA Synthesis kit (cat. no. K1622; Thermo Fisher Scientific, Inc.) according to the manufacturer's instructions. qPCR was performed using SYBR Green I (Invitrogen; Thermo Fisher Scientific, Inc.) and the CFX96 ${ }^{\mathrm{TM}}$ Real-Time System (Bio-Rad Laboratories, Inc., Hercules, CA, USA). Target miRNA expression levels were quantified relative to U6 expression levels. qPCR was performed in a reaction volume of $20 \mu \mathrm{l}$ consisting of $10 \mu \mathrm{l}$ SYBR green mix (Beijing Novogene Bioinformatics Technology Co., Ltd., Beijing, China), $2 \mu \mathrm{l}$ cDNA, $0.8 \mu \mathrm{l}$ primers and $7.2 \mu \mathrm{l}$ diethylpyrocarbonate double-distilled $\mathrm{H}_{2} \mathrm{O}$. Reaction mixtures were incubated in eight-strip tubes for $10 \mathrm{~min}$ at $95^{\circ} \mathrm{C}$, followed by 40 cycles of $90^{\circ} \mathrm{C}$ for $5 \mathrm{sec}, 60^{\circ} \mathrm{C}$ for $30 \mathrm{sec}$ and $4^{\circ} \mathrm{C}$ indefinitely. Target miRNA levels were quantified using the $2^{-\Delta \Delta \mathrm{Cq}}$ method (11). For each target gene, samples were analyzed in triplicate.

Target prediction and function analysis. GO terms (www. geneontology.org) and the Kyoto Encyclopedia of Genes and Genomes (KEGG; www.genome.jp/kegg/) pathway annotation databases were employed to identify potential miRNA target genes and provide an insight into the function of miRNAs. $\mathrm{GO}$ analysis was conducted to organize genes into hierarchical categories and determine the miR-gene regulatory network (12). GOs with a P-value of $<0.001$ and with a FDR of $<0.05$ were selected Fisher's exact test was then used to provide the P-value for each GO term, which represented the probability that the observed counts may have been due to chance. In addition, pathway analysis was utilized to identify potential pathways of the differentially expressed genes, using the KEGG database. In order to identify enriched pathways, Fisher's exact test and the $\chi^{2}$-test were performed, and the threshold for significance was defined by $\mathrm{P}<0.001$ and $\mathrm{FDR}<0.05(13,14)$.

miRNA-gene network analysis. Differentially expressed miRNA target genes in significant GO and pathway categories 
Table I. Primer sequences.

\begin{tabular}{lll}
\hline Gene ID & \multicolumn{1}{c}{ Primer } & \multicolumn{1}{c}{ Sequence $\left(5^{\prime} \rightarrow 3^{\prime}\right)$} \\
\hline rno-miR-212-3p & rno-miR-212-3p-R & CTCAACTGGTGTCGTGGAGTCGGCAATTCAGTTGAGTGGCCGTG \\
& rno-miR-212-3p-F & TAACAGTCTCCAGTCACGGCCA \\
rno-miR-212-5p & rno-miR-212-5p-R & CTCAACTGGTGTCGTGGAGTCGGCAATTCAGTTGAGCAGTAAGC \\
& rno-miR-212-5p-F & GGACCTTGGCTCTAGACTGCTTACTG \\
rno-miR-34c-5p & rno-miR-34c-5p-R & CTCAACTGGTGTCGTGGAGTCGGCAATTCAGTTGAGGCAATCAG \\
& rno-miR-34c-5p-F & GGAGGCAGTGTAGTTAGCTGATTGC \\
rno-miR-369-5p & rno-miR-369-5p-R & CTCAACTGGTGTCGTGGAGTCGGCAATTCAGTTGAGGCGAATAT \\
& rno-miR-369-5p-F & CTGGGAGATCGACCGTGTTATATTCGC \\
rno-miR-187-3p & rno-miR-187-3p-R & CTCAACTGGTGTCGTGGAGTCGGCAATTCAGTTGAGCCGGCTGC \\
& rno-miR-187-3p-F & TCGTGTCTTGTGTTGCAGCCGG \\
rno-miR-672-3p & rno-miR-672-3p-R & CTCAACTGGTGTCGTGGAGTCGGCAATTCAGTTGAGTCGAAGAT \\
& rno-miR-672-3p-F & GGACACACAGTCGCCATCTTCGA \\
rno-miR-141-5p & rno-miR-141-5p-R & CTCAACTGGTGTCGTGGAGTCGGCAATTCAGTTGAGCAACACTG \\
& rno-miR-141-5p-F & CTGGGTCCATCTTCCAGTGCAGTGTTG \\
U6 & U6-R & CGCTTCACGAATTTGCGTGTCAT \\
& U6-F & GCTTCGGCAGCACATATACTAAAAT
\end{tabular}

Rno, Rattus norvegicus; miR, microRNA; R, reverse; F, forward.
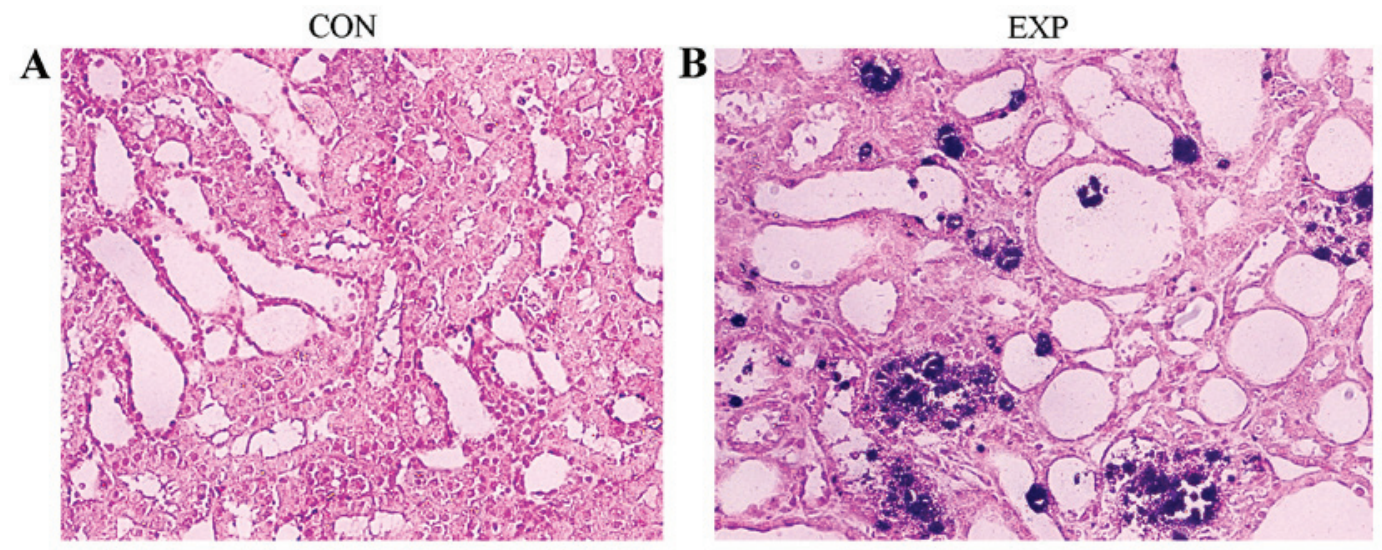

Figure 1. Examination of kidney tissue sections by light microscopy following von Kossa staining. Microscope images of kidney tissues derived from (A) CON and (B) EXP rats. Numerous calcium crystals (stained black-brown with argent nitrate) can be observed in Fig. 1B (magnification, x200). CON, rats provided with water for 2 weeks; EXP, rats provided with water containing $1.0 \%$ ethylene glycol and $0.5 \%$ ammonium chloride for 2 weeks to induce hyperoxaluria.

were analyzed using miRNA target gene network analysis. In order to establish a miRNA-gene network, the association between miRNAs and potential target genes was determined by their differential expression values and predicted interactions using the Sanger miRBase database (http://www.mirbase. org/). If a potential correlation between a miRNA and specific target genes existed, the results were classified as 'a miRNA targets a gene' or 'a gene contains a predicted binding site for a miRNA'. 'Degree' represented the association between 'a miRNA and target genes' or 'a gene and miRNAs' in the network. Briefly, a high degree indicated a more important role in the network. The center of the network was represented by the degree of contribution of one miRNA to the surrounding genes or the contribution of one gene to the surrounding miRNAs. Critical miRNAs in the network invariably had the highest degrees (15-17).

\section{Results}

Renal tissue samples from the CON group indicated higher levels of oxalate. As presented in Fig. 1, the renal tissues derived from rats in the EXP group were pale with numerous visible calcium oxalate deposits. Rat renal tissues from EXP and CON groups were stained using the von Kossa method and calculus crystals were stained brown-black. Microscopic examination revealed that crystals were more abundant in the EXP group compared with the CON group (Fig. 1). In addition, rats from the EXP group exhibited significantly higher levels of oxalate in the urine compared with those from the CON group $(\mathrm{P}<0.01$; Fig. 2$)$.

The miRNA expression profiles in the renal tissues of hyperoxaluric rats were altered. Hyperoxaluric rats following 
treatment with $1 \%$ ethylene glycol and $0.5 \%$ ammonium chloride, exhibited markedly altered expression profiles of miRNA in renal tissues compared with the normal controls (Fig. 3). A total of 28 miRNAs were differentially expressed, with a $>2$-fold change in expression between EXP and CON groups (Table II). Out of these, 20 miRNAs were upregulated $(>0.5$-2-fold change) and 8 miRNAs were downregulated ( $\leq 0.5$-fold change). The results of the hierarchical cluster analysis of differentially expressed miRNAs between EXP and CON groups are presented in Fig. 3 .

RT-qPCR analysis verified the array results. In order to validate the results obtained from the miRNA microarray analysis, RT-qPCR analysis was conducted to determine the expression of five differentially expressed miRNAs, including miR-214-3p, miR-146b-5p, miR-31a-5p, miR-369-5p and miR-141-5p, and the results from microarray and RT-qPCR were subsequently compared. RT-qPCR analysis demonstrated that miR-214-3p, miR-146b-5p, miR-31a-5p were upregulated, while miR-369-5p and miR-141-5p were downregulated. These results were comparable to those obtained by microarray analysis (Table II).

GO and pathway analysis demonstrated miRNAs were involved in numerous functions. The GO threshold of significance was defined as $\mathrm{P}<0.001$ and $\mathrm{FDR}<0.05$. A total of 1,070 $\mathrm{GO}$ enriched terms were identified as predicted functions of overexpressed miRNAs and included positive regulation of cell proliferation, cytokine production, and apoptotic processes. By contrast, the 863 significant GO terms that were associated with downregulated miRNAs included intracellular signal transduction and regulation of cell proliferation. The significant functions and pathways are presented in Figs. 5 and 6 . According to the target analysis, a number of critical biological functions, including intracellular signal transduction, regulation of apoptotic processes and regulation of cell proliferation, were observed to involve miRNAs. Furthermore, the results of the pathway analysis suggest that these miRNAs serve important roles in distinct biological processes, such as the insulin signaling, mitogen-activated protein kinase (MAPK) and phosphatidylinositol-3 kinase (PI3K)/Akt serine-threonine kinase 1 (AKT) signaling pathways.

miRNA-gene network analysis demonstrated 12 key miRNAs. A total of 12 miRNAs (Table III) demonstrated a high degree score, which represents an important role in the miRNA-gene network. These miRNAs were predicted to regulate Lipin 2, which is a metabolism-associated gene, as well as and 17 additional genes [degree $\geq 4$; potassium inwardly-rectifying channel, subfamily J, member 6 (Kcnj6), Tnf receptor-associated factor 3 (Traf3), transducin $(\beta)$-like $1 \mathrm{X}$-linked receptor 1 , serine/threonine kinase 4, Sp1 transcription factor, Dishevelled, dsh homolog 1, Lim domains containing 1, transforming growth factor- $\alpha$, Elk4, ETS-domain protein (SRF accessory protein 1), chemokine (C-X-C motif) ligand 12, adenosylmethionine decarboxylase 1, Slingshot protein phosphatase 2, cannabinoid receptor 2 (macrophage), inhibin $\beta$-B, glutamate receptor, ionotropic, AMPA 2, and laminin $\gamma 3$; Table IV]. Among these genes, Kcnj6 was the most highly regulated target gene.

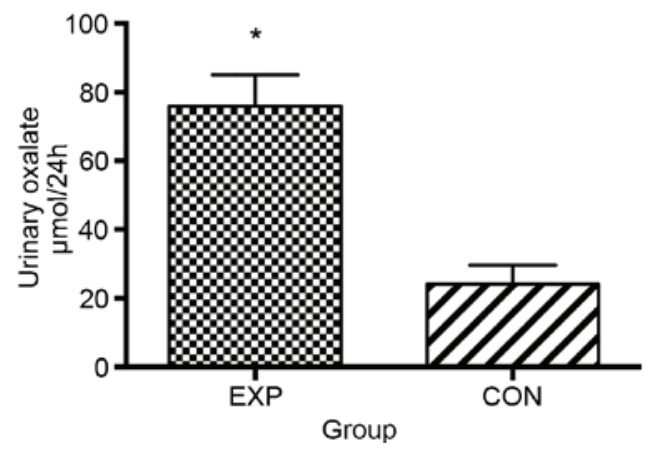

Figure 2. Ethylene glycol and ammonium chloride increases rat urinary oxalate levels. Urine specimens were collected (in triplicate) at $24 \mathrm{~h}$-intervals from rats in the EXP and CON groups and oxalate concentrations were determined on day 14 using ion chromatography. The mean \pm standard deviation urine oxalate concentration in CON and EXP groups were 24.16 \pm 1.727 and $75.91 \pm 2.885 \mu \mathrm{mol} / 24 \mathrm{~h}$, respectively. ${ }^{*} \mathrm{P}<0.01$ vs. CON group. miRNA, microRNA; EXP, rats provided with water containing $1.0 \%$ ethylene glycol and $0.5 \%$ ammonium chloride for 2 weeks to induce hyperoxaluria; CON, rats provided with water for 2 weeks.

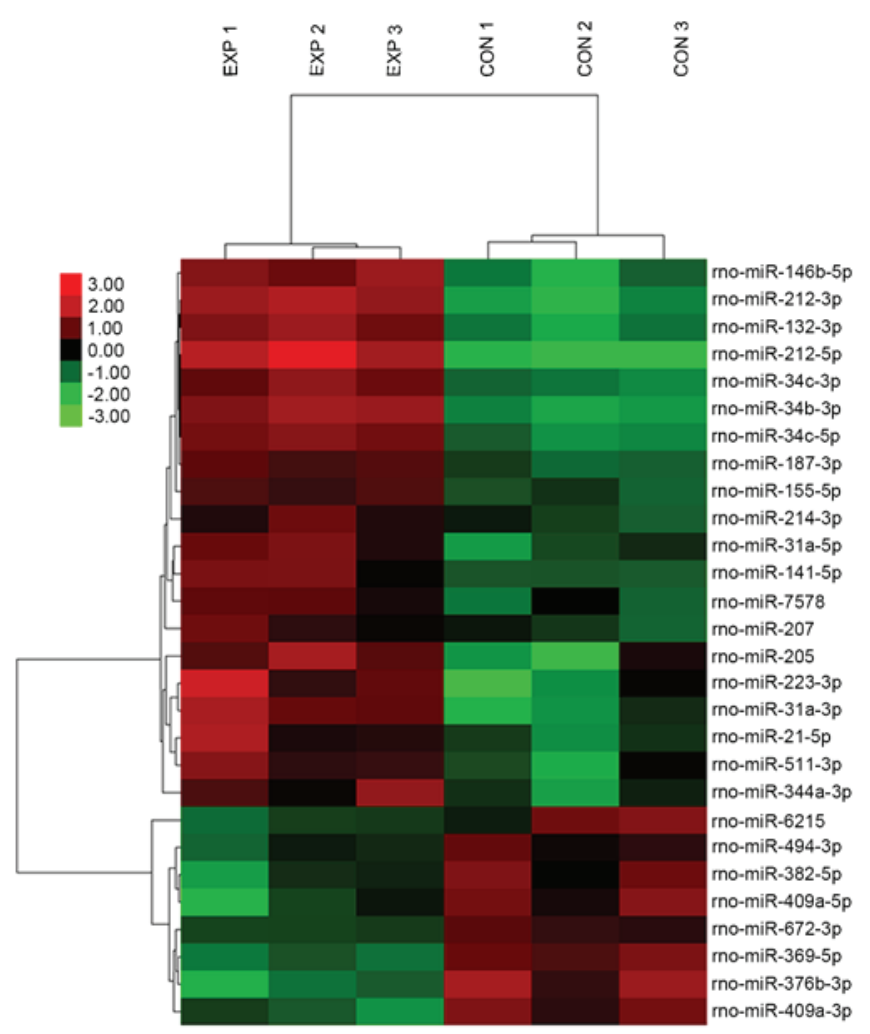

Figure 3. Hierarchical cluster heat map of differentially expressed miRNAs in rat renal tissues from EXP and CON groups. Red signals indicate downregulated expression and green signals indicate upregulated expression. EXP, rats provided with water containing $1.0 \%$ ethylene glycol and $0.5 \%$ ammonium chloride for 2 weeks to induce hyperoxaluria; $\mathrm{CON}$, rats provided with water for 2 weeks.

\section{Discussion}

Urolithiasis is a multifactorial disease that comprises a large proportion of urinary tract diseases. A number of hypotheses have been investigated in previous studies in order to understand the mechanisms of oxalate stone formation (18-21). The results demonstrate that stone formation may be the result of 
Table II. miRNAs that were differentially expressed with a $>2$-fold change.

\begin{tabular}{lc}
\hline Probe ID & Fold-change $(\mathrm{EXP} / \mathrm{CON})$ \\
\hline Upregulated & \\
rno-miR-212-5p & 15.54 \\
rno-miR-212-3p & 8.75 \\
rno-miR-34b-3p & 7.28 \\
rno-miR-132-3p & 5.82 \\
rno-miR-146b-5p & 5.77 \\
rno-miR-223-3p & 5.28 \\
rno-miR-31a-3p & 5.19 \\
rno-miR-34c-5p & 5.02 \\
rno-miR-34c-3p & 4.64 \\
rno-miR-205 & 4.56 \\
rno-miR-31a-5p & 3.19 \\
rno-miR-187-3p & 3.05 \\
rno-miR-141-5p & 3.01 \\
rno-miR-21-5p & 2.96 \\
rno-miR-511-3p & 2.92 \\
rno-miR-344a-3p & 2.83 \\
rno-miR-155-5p & 2.66 \\
rno-miR-7578 & 2.54 \\
rno-miR-214-3p & 2.17 \\
rno-miR-207 & 2.10 \\
Downregulated & \\
rno-miR-494-3p & 0.48 \\
rno-miR-672-3p & 0.43 \\
rno-miR-6215 & 0.37 \\
rno-miR-382-5p & 0.35 \\
rno-miR-409a-5p & 0.30 \\
rno-miR-409a-3p & 0.27 \\
\hline
\end{tabular}

Rno, Rattus norvegicus; miR, microRNA; CON, rats provided with water for 2 weeks; EXP, rats provided with water containing $1.0 \%$ ethylene glycol and $0.5 \%$ ammonium chloride for 2 weeks to induce hyperoxaluria.

interactions between epithelial cells, matrix cells and macrophages, as well as additional cell types, in a hyperoxaluric microenvironment. Thus, the aim of the present study was to investigate whether alterations in miRNA expression occur in an in vivo model of hyperoxaluria are a potential process that underlies calcium oxalate stone formation. Ethylene glycol and ammonium chloride, which are typically used to generate rat models of hyperoxaluria (22), were used to induce crystal stone formation. In a previous study, a stable experimental model was established using $1 \%(\mathrm{v} / \mathrm{v})$ ethylene glycol and $0.5 \%$ ammonium chloride (23).

Previous studies have demonstrated that kidney stones form as a result of interactions between genetic and environmental factors $(24,25)$. High concentrations of urine oxalate may result in tubular epithelial cell injury, which may
Table III. Key miRNAs (degree $\geq 59$ ) in the miRNA target gene network.

\begin{tabular}{lcc}
\hline Gene symbol & Degree & Regulation \\
\hline rno-miR-207 & 202 & $\mathrm{Up}$ \\
rno-miR-214-3p & 179 & $\mathrm{Up}$ \\
rno-miR-212-5p & 131 & $\mathrm{Up}$ \\
rno-miR-34c-5p & 129 & $\mathrm{Up}$ \\
rno-miR-7578 & 95 & $\mathrm{Up}$ \\
rno-miR-672-3p & 92 & Down \\
rno-miR-205 & 75 & $\mathrm{Up}$ \\
rno-miR-141-5p & 75 & $\mathrm{Up}$ \\
rno-miR-146b-5p & 72 & $\mathrm{Up}$ \\
rno-miR-212-3p & 68 & $\mathrm{Up}$ \\
rno-miR-31a-5p & 65 & $\mathrm{Up}$ \\
rno-miR-187-3p & 59 & $\mathrm{Up}$ \\
\hline
\end{tabular}

Degree represents the number of genes that are regulated by the miRNA. A higher degree indicates that a greater number of genes are regulated by the miRNA. Regulation indicates whether the miRNA was upregulated or downregulated. Rno, Rattus norvegicus; miR, microRNA.

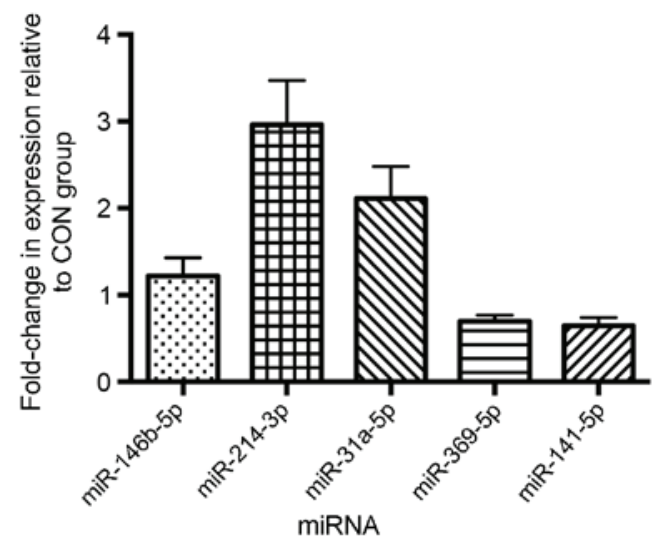

Figure 4. Reverse transcription-quantitative polymerase chain reaction analysis validating the expression of five differentially expressed miRNAs identified by microarray analysis of rat renal tissues from CON and EXP groups. The selected miRNAs consisted of three upregulated miRNAs and two downregulated miRNAs in rat renal tissues from the EXP group relative to the CON group. miRNA, microRNA; CON, rats provided with water for 2 weeks; EXP, rats provided with water containing $1.0 \%$ ethylene glycol and $0.5 \%$ ammonium chloride for 2 weeks to induce hyperoxaluria.

subsequently induce gene expression and protein synthesis, ultimately leading to nephrolithiasis $(26,27)$. In order to elucidate this process, numerous in vivo and in vitro studies have investigated the role of genes, mRNA and proteins in the pathogenesis of hyperoxaluria (28-30). These studies demonstrated that PAT1, also known as solute carrier family 26, member 1 , is important in oxalate transportation and that p38 MAPK mediates calcium oxalate crystal-induced disruption of the distal renal tubular epithelial tight junctions (31-33). However, only a limited number of studies have investigated the role of miRNAs in the pathogenesis of hyperoxaluria and urolithiasis. 
Predicted GO functions of genes targeted by differentially expressed miRNAs

\author{
Intracellular signal transduction
Small GTPase mediated signal transduction
Negative regulation of cell proliferation \\ Protein transport \\ Signal transduction \\ Positive regulation of cell proliferation
}

Positive regulation of transcription from RNA polymerase II promoter

Negative regulation of transcription, DNA-dependent

Response to drug

Negative regulation of transcription from RNA polymerase II promoter

Positive regulation of transcription, DNA-dependent

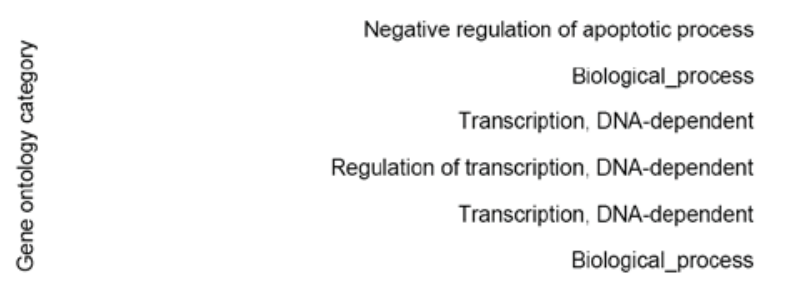

Negative regulation of transcription from RNA polymerase II promoter

Positive regulation of transcription, DNA-dependent

Regulation of transcription, DNA-dependent

Negative regulation of apoptotic process

Negative regulation of transcription, DNA-dependent

Protein phosphorylation

Positive regulation of transcription from RNA polymerase II promoter

Response to drug

Positive regulation of cell proliferation

Negative regulation of cell proliferation

Protein transport

Small GTPase mediated signal transduction

Intracellular signal transduction
Enrichment

\begin{tabular}{lllllll}
-6 & -4 & -2 & 0 & 2 & 4 & 6 \\
\hline
\end{tabular}

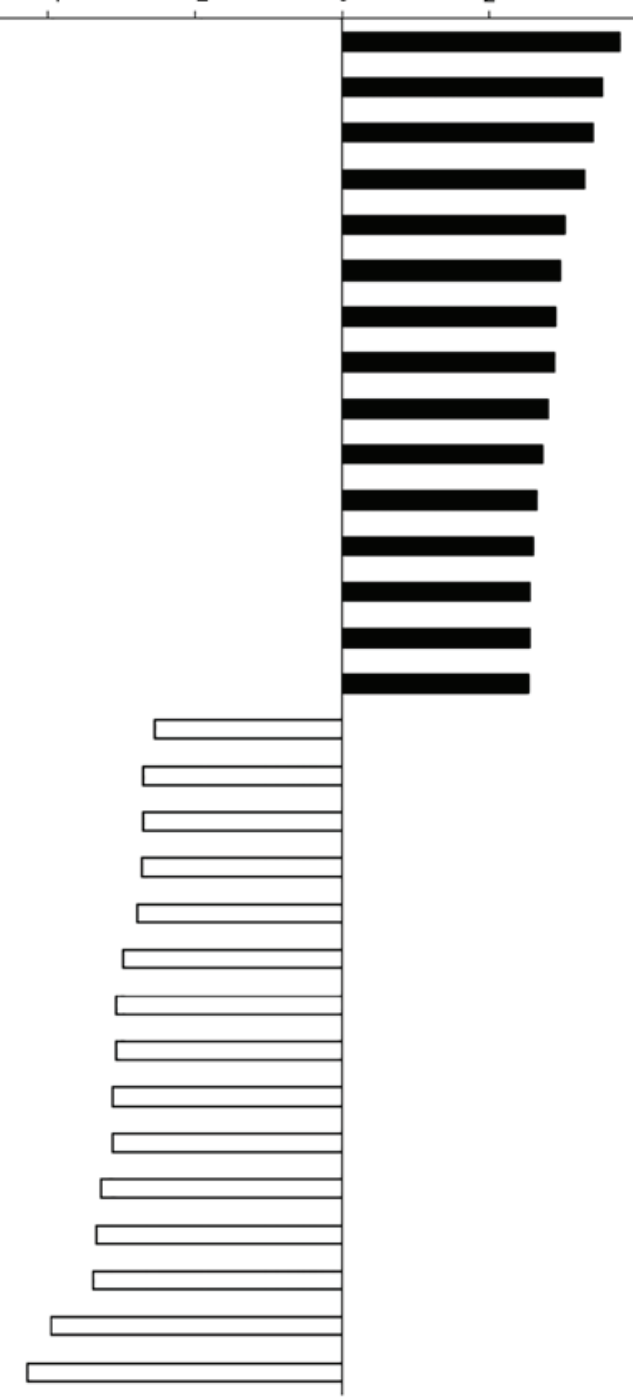

Figure 5. Significant GO functional maps of genes targeted by differentially expressed miRNAs identified following microarray analysis of rat renal tissues from CON and EXP groups. The GO category and enrichment of GOs is presented on the vertical and horizontal axes, respectively. A higher number indicates that the function is more significant. GO, gene ontology; miRNA, microRNA; CON, rats provided with water for 2 weeks; EXP, rats provided with water containing $1.0 \%$ ethylene glycol and $0.5 \%$ ammonium chloride for 2 weeks to induce hyperoxaluria.

Wang et al (28) demonstrated that 25 miRNAs were differentially expressed in the HK-2 immortalized proximal tubule epithelial cell line following exposure to oxalate monohydrate crystals, and the majority of these were associated with apoptosis and mitochondrial and metabolic processes. In order to investigate the upregulation and downregulation of miRNAs in vivo in the present study, the miRNA expression profiles of hyperoxaluric rats were examined and compared with wild-type controls, and 28 differentially expressed miRNAs were identified. These differentially expressed miRNAs were associated with multiple biological processes, including response to insulin, apoptosis, intracellular signaling cascade and the inflammatory response. RT-qPCR analysis of miRNA levels between wild-type rats and those with hypoxaluria were largely consistent with the microarray analysis. In order to identify potential target genes and the functions of differentially expressed miRNAs, GO and pathway analyses were performed following microarray analysis. The results demonstrated that 1,059 genes were predicted to be targets of the 28 identified miRNAs, which were differentially expressed with a $>2$-fold change. In addition, miRNA-target gene-network analyses were used to determine the degree of interaction between miRNAs and predicted target genes. To generate a miRNA-target gene-network diagram, the putative association between miRNAs and target genes was determined by their differential expression values, and based on their interactions according to the Sanger miRNA database. The results revealed 17 highly regulated target genes and 12 key miRNAs in the miRNA target gene network. The most highly regulated target gene was Kcnj6, which encodes a potassium channel that belongs to subfamily J. Rno-miR-214-3p, rno-miR-212-5p and rno-miR-7578 were associated with multiple biological processes, including the regulation of apoptosis, negative regulation of nuclear factor- $\kappa \mathrm{B}(\mathrm{NF}-\kappa \mathrm{B})$ transcription factor 
Table IV. Highest degree of target genes in the miRNA target gene network (degree $\geq 4$ ).

Gene symbol

Definition

Degree

Kcnj6

Traf3

Tbl1xr1

Stk4

Lpin2

Sp1

Dv11

Limd1

Tgfa

Elk4

Cxcl12

Amd1

Ssh2

Cnr2

Inhbb

Gria2

Lamc3
Potassium inwardly-rectifying channel, subfamily J, member 6 7

Tnf receptor-associated factor 3

Transducin $(\beta)$-like $1 \mathrm{X}$-linked receptor 1

Serine/threonine kinase 4

Lipin 2

$\mathrm{Sp} 1$ transcription factor

Dishevelled, dsh homolog 1 (Drosophila)

LIM domains containing 1

Transforming growth factor- $\alpha$

ELK4, ETS-domain protein (SRF accessory protein 1)

Chemokine (C-X-C motif) ligand 12

Adenosylmethionine decarboxylase 1

Slingshot protein phosphatase 2

Cannabinoid receptor 2 (macrophage)

Inhibin $\beta$-B

Glutamate receptor, ionotropic, AMPA 2

Laminin $\gamma 3$
5

5

5

4

4

4

4

4

4

4

4

4

4

4

4

4

Degree represents the impact of one miRNA to the surrounding genes or the impact of one gene to the surrounding miRNAs. A higher degree indicates greater regulation by miRNAs.

A

A

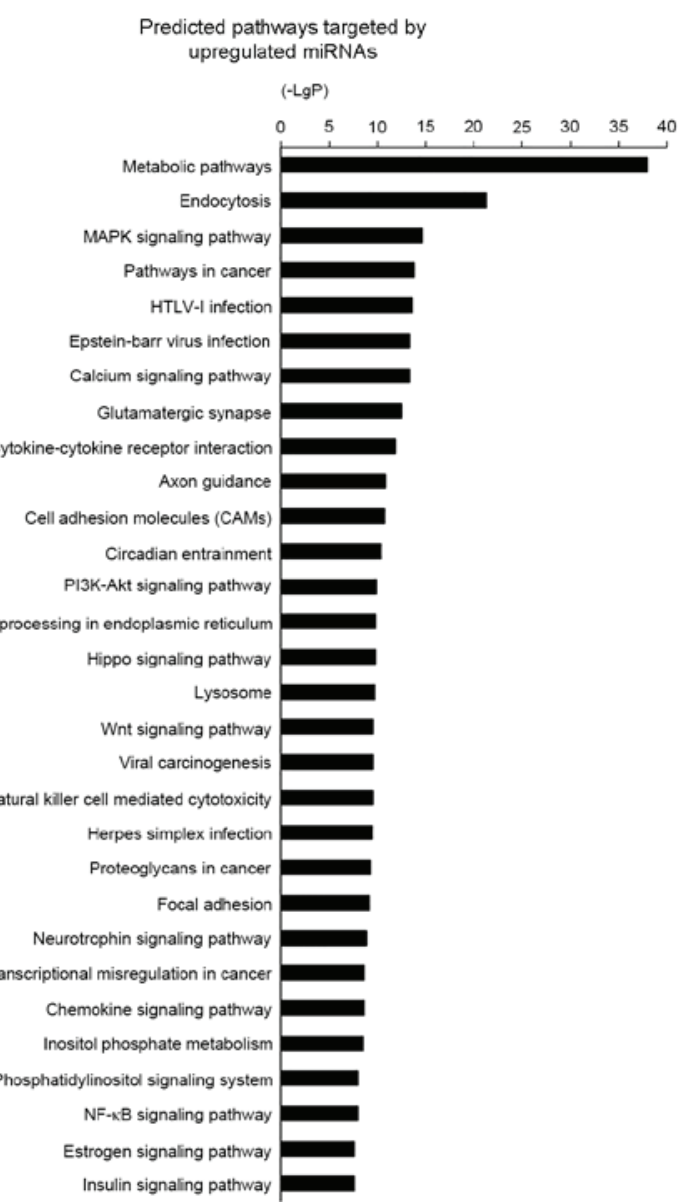

B Predicted pathways targeted by
downregulated miRNAs

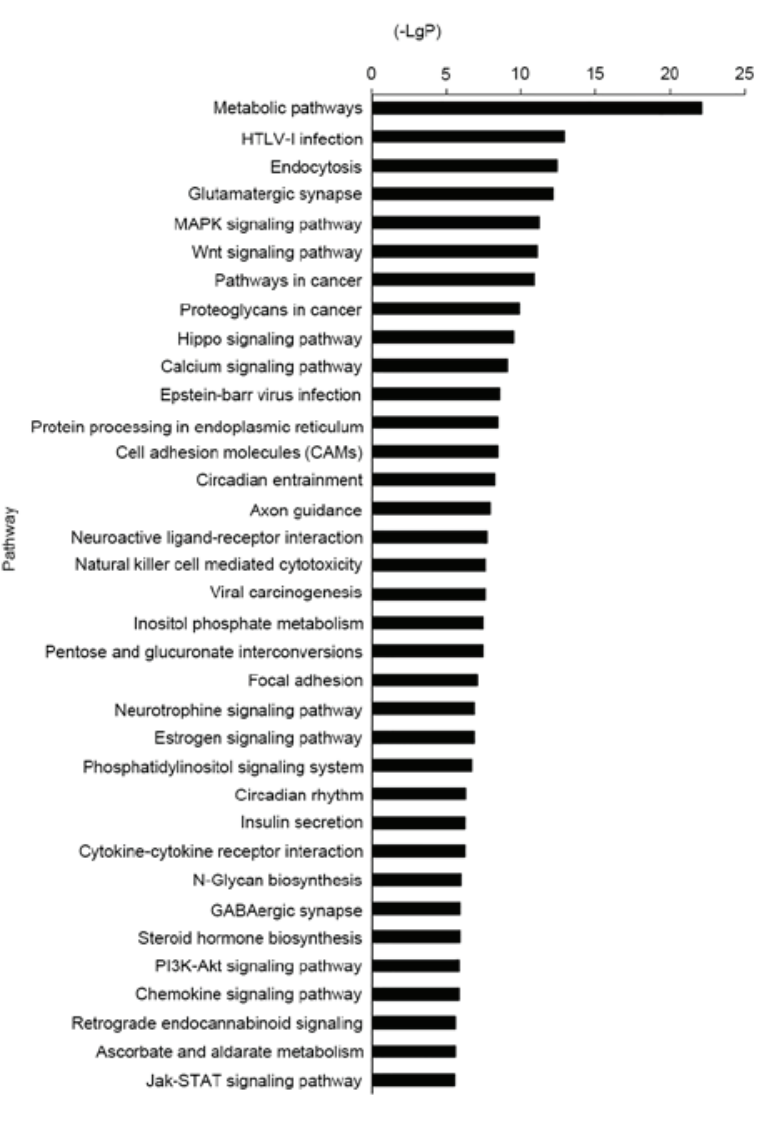

Figure 6. Pathway analysis of genes targeted by differentially expressed miRNAs identified following microarray analysis of rat renal tissues from CON and EXP groups. Significant pathways targeted by (A) upregulated and (B) downregulated miRNAs. Enrichment scores of biological processes are indicated by-log (P-value). miRNA, microRNA; CON, rats provided with water for 2 weeks; EXP, rats provided with water containing $1.0 \%$ ethylene glycol and $0.5 \%$ ammonium chloride for 2 weeks to induce hyperoxaluria. 
activity, the innate immune response, regulation of cytokine production, and the Toll-like receptor signaling pathway. In addition, rno-miR-7578, rno-miR-141-5p, rno-miR-146b-5p and rno-miR-187-3p were associated with the positive regulation of transcription via the RNA polymerase II promoter, protein dephosphorylation and lipid metabolism. Furthermore, rno-miR-141-5p, rno-miR-207, rno-miR-34c-5p and rno-miR-31a-5p were associated with the positive regulation of cell proliferation and the regulation of calcium ion transport.

According to the GO analysis, rno-miR-132-3p, rno-miR-146b-5p, rno-miR-187-3p, rno-miR-205, rnomiR-207, rno-miR-212-3p, rno-miR-214-3p, rno-miR-21-5p and rno-miR-34b-3p were predicted to be associated with the upregulation of the protein kinase B signaling cascade, whereas rno-miR-214-3p, rno-miR-212-5p, rno-miR-7578 and rno-miR-207 were predicted to be involved in the downregulation of NF-кB transcription factor activity. Traf3 is important role in cell function and in the upregulation of $\mathrm{NF}-\mathrm{KB}$ transcription factor-associated genes, the regulation of biological and apoptotic processes, the negative regulation of $\mathrm{NF}-\mathrm{\kappa B}$ transcription factor activity, the regulation of cytokine production, and the Toll-like receptor-signaling pathway. Several studies have implicated interleukin-1 in calcium oxalate stone formation through the stimulation of multiple TLR ligands, and Oganesyan et al (35) demonstrated that a Traf3-null cell line is unable to produce interleukin-1 (34-37). In the present study, induction of apoptosis was predicted to be an important feature that involves rno-miR-132-3p, rno-miR-146b-5p, rno-miR-187-3p, rno-miR-205, rno-miR-207, rno-miR-212-3p, rno-miR-214-3p, rno-miR-21-5p, rno-miR-223-3p and rno-miR-34c-5p. Interestingly, Fujii et al (38) reported that the preventive effect of adiponectin treatment in kidney crystal formation occurs due to the inhibition of inflammation and apoptosis.

Pathway analysis revealed that the differentially expressed miRNAs identified in the present study may serve a role in multiple crucial pathways, including porphyrin and chlorophyll metabolism, the PI3K-AKT signaling pathway, insulin secretion and the insulin signaling pathway. The PI3K-AKT signaling pathway is associated with metabolic syndrome, and it has been reported that PI-3Kp85 mRNA expression decreased in rats with metabolic syndrome (39). Metabolic syndrome is a disorder of energy utilization and storage, which is characterized by high serum triglycerides, elevated fasting plasma glucose, abdominal obesity, elevated blood pressure, and low high-density lipoprotein cholesterol levels (40). In previous studies, urolithiasis was demonstrated to be associated with a history of metabolic syndrome and specific metabolic syndrome traits, particularly dyslipidemia [odds ratio $(\mathrm{OR})=1.36]$, and any one of the five metabolic syndrome traits was associated with increased oxalate excretion $(\mathrm{OR}=2.10)(41,42)$. Kabeya et al $(43)$ recently demonstrated that a fasting plasma glucose level of $\geq 126 \mathrm{mg} / \mathrm{dl}$ was significantly associated with a risk of kidney stones $(\mathrm{OR}=1.83)$. However, it remains unknown how kidney stone disease is connected with metabolic syndrome. A recent study suggested that the production of reactive oxygen species, and the progression of oxidative stress and inflammation may be associated with these shared pathways (44). In the present study, differentially expressed miRNAs were predicted to be involved in insulin and PI3K-AKT signaling pathways as indicated by the pathway analysis. However, additional research is needed to investigate the molecular mechanism of hyperoxaluria further.

To the best of our knowledge, the present study is the first to investigate the role of miRNA expression profiles in calcium oxalate stone formation in vivo. The differentially expressed miRNAs identified in the kidney tissues of rats with hyperoxaluria, may provide a novel perspective on the pathogenic mechanisms of kidney stone formation. However, it should be noted that even though the miRNAs were differentially expressed in this setting, not all miRNAs may serve a key role in kidney stone formation. In addition, the miRNAs identified in rat kidney tissues may not be involved in the same process in humans. Therefore, miRNA and targeted gene studies should be performed in human cells or samples to confirm the results of the current study.

In conclusion, the expression of multiple miRNAs was demonstrated to be altered in the kidneys of rats with ethylene glycol-induced hyperoxaluria. Thus, the mechanistic pathways of hyperoxaluria in kidneys may be due to alterations in the expression of several of these identified miRNAs. Furthermore, the functional significance of these expression alterations in hyperoxaluria remains unknown. Future studies should aim to clarify this and determine the adaptive responses that occur in tissues during hyperoxaluria, which may lead to a better understanding of the molecular mechanisms of urolithiasis.

\section{Acknowledgements}

The current study was supported by the National Natural Science Foundation of China (grant no. 81400706).

\section{References}

1. Coe FL, Evan A and Worcester E: Kidney stone disease. J Clin Invest 115: 2598-2608, 2005.

2. Ambros V: The functions of animal microRNAs. Nature 431: 350-355, 2004.

3. Bartel DP: MicroRNAs: Genomics, biogenesis, mechanism, and function. Cell 116: 281-297, 2004.

4. Lewis BP, Burge CB and Bartel DP: Conserved seed pairing, often flanked by adenosines, indicates that thousands of human genes are microRNA targets. Cell 120: 15-20, 2005.

5. Lewis BP, Shih IH, Jones-Rhoades MW, Bartel DP and Burge CB: Prediction of mammalian microRNA targets. Cell 115: 787-798, 2003.

6. Xuehua C, Xiaojian G, Xizhao S, Li Z and Yan X: The study of the determination of oxalate and citrate in human urine by lon chromatography method. J Clin Urology (China) 28: 437-439, 2013.

7. Institute of Laboratory Animal Resources (US). Committee on Care, Use of Laboratory Animals, National Institutes of Health (US). Division of Research Resources. Guide for the care and use of laboratory animals[M]. National Academies, 1985.

8. Wright GW and Simon RM: A random variance model for detection of differential gene expression in small microarray experiments. Bioinformatics 19: 2448-2455, 2003.

9. Bolstad BM: Low-level analysis of high-density oligonucleotide array data: Background, normalization and summarization. University of California, Berkeley, 2004.

10. Yang H, Crawford N, Lukes L, Finney R, Lancaster M and Hunter KW: Metastasis predictive signature profiles pre-exist in normal tissues. Clin Exp Metastasis 22: 593-603, 2005.

11. Livak KJ and Schmittgen TD: Analysis of relative gene expression data using real-time quantitative PCR and the 2(-Delta Delta C(T)) method. Methods 25: 402-408, 2001.

12. Gene Ontology Consortium: The gene ontology (GO) project in 2006. Nucleic Acids Res 34 (Database Issue): D322-D326, 2006. 
13. Kanehisa M, Goto S, Kawashima S, Okuno Y and Hattori M: The KEGG resource for deciphering the genome. Nucleic Acids Res 32 (Database Issue): D277-D280, 2004.

14. Yi M, Horton JD, Cohen JC, Hobbs HH and Stephens RM: WholePathwayScope: A comprehensive pathway-based analysis tool for high-throughput data. BMC Bioinformatics 7: 30, 2006.

15. Enright AJ, John B, Gaul U, Tuschl T, Sander C and Marks DS: MicroRNA targets in drosophila. Genome Biol 5: R1, 2003.

16. Joung JG, Hwang KB, Nam JW, Kim SJ and Zhang BT: Discovery of microRNA-mRNA modules via population-based probabilistic learning. Bioinformatics 23: 1141-1447, 2007.

17. Shalgi R, Lieber D, Oren M and Pilpel Y: Global and local architecture of the mammalian microRNA-transcription factor regulatory network. PLoS Comput Biol 3: e131, 2007.

18. Evan AP, Coe FL, Lingeman JE, Shao Y, Matlaga BR, Kim SC, Bledsoe SB, Sommer AJ, Grynpas M, Philips CL and Worcester EM: Renal crystal deposits and histopathology in patients with cystine stones. Kidney Int 69: 2227-2235, 2006.

19. Evan AP, Lingeman JE, Coe FL, Parks JH, Bledsoe SB, Shao Y, Sommer AJ, Patterson RF, Kuo RL and Grynpas M: Randall plaque of patients with nephrolithisis begins in basement membranes of thin loops of Henle. J Clin Invest 111: 607-616, 2003.

20. Verkoelen CF and Verhulst A: Proposed mechanisms in renal tubular crystal retention. Kidney Int 72: 13-18, 2007.

21. Taylor ER and Stoller ML: Vascular theory of the formation of Randall plaques. Urolithiasis 43 (Suppl 1): S41-S45, 2015.

22. de Bruijn WC, Boevé ER, van Run PR, van Miert PP, de Water R, Romijn JC, Verkoelen CF, Cao LC and Schröder FH: Etiology of calcium oxalate nephrolithiasis in rats. I. Can this be a mode for human stone formation? Scanning Microsc 9: 103-114, 1995.

23. Cao Z and Liu J: Comparison of several experimental renal calcium oxalate Calcalus ModelS in rats. J Huazhong Univ Sci Tech 31: 556-559, 2002.

24. Wisener LV, Pearl DL, Houston DM, Reid-Smith RJ and Moore AE: Risk factors for the incidence of calcium oxalate uroliths or magnesium ammonium phosphate uroliths for dogs in Ontario, Canada, from 1998 to 2006. Am J Vet Res 71: 1045-1054, 2010.

25. Halbritter J, Baum M, Hynes AM, Rice SJ, Thwaites DT, Gucev ZS, Fisher B, Spaneas L, Porath JD, Braun DA, et al: Fourteen monogenic genes account for $15 \%$ of nephrolithiasis nephrocalcinosis. J Am Soc Nephrol 26: 543-551, 2015.

26. Ouyang JM, Yao XQ, Tan J and Wang FX: Renal epithelial cell injury and its promoting role in formation of calcium oxalate monohydrate. J Biol Inorg Chem 16: 405-416, 2011.

27. Sivalingam S, Nakada SY, Sehgal PD, Crenshaw TD and Penniston KL: Dietary hydroxyproline induced calcium oxalate lithiasis and associated renal injury in the porcine model J Endourol 27: 1493-1498, 2013.

28. Wang B, Wu B, Liu J, Yao W, Xia D, Li L, Chen Z, Ye Z and Yu X Analysis of altered microRNA expression profiles in proximal renal tubular cells in response to calcium oxalate monohydrate crystal adhesion: Implications for kidney stone disease. PLoS One 9: e101306, 2014.

29. Okumura N, Tsujihata M, Momohara C, Yoshioka I, Suto K, Nonomura N, Okuyama A and Takao T: Diversity in protein profiles of individual calcium oxalate kidney stones. PLoS One 8: e68624, 2013.
30. Halbritter J, Baum M, Hynes AM, Rice SJ, Thwaites DT, Gucev ZS, Fisher B, Spaneas L, Porath JD, Braun DA, et al: Fourteen monogenic genes account for $15 \%$ of nephrolithiasis/nephrocalcinosis. J Am Soc Nephrol 26: 543-551, 2015.

31. Jiang Z, Asplin JR, Evan AP, Rajendran VM, Velazquez H, Nottoli TP, Binder HJ and Aronson PS: Calcium oxalate urolithiasis in mice lacking anion transporter Slc26a6. Nat Genet 38: 474-478, 2006.

32. Corbetta S, Eller-Vainicher C, Frigerio M, Valaperta R, Costa E, Vicentini L, Baccarelli A, Beck-Peccoz P and Spada A: Analysis of the 206M polymorphic variant of the SLC26A6 gene encoding a Cl-oxalate transporter in patients with primary hyperparathyroidism. Eur J Endocrinol 160: 283-288, 2009.

33. Alper SL and Sharma AK: The SLC26 gene family of anion transporters and channels. Mol Aspects Med 34: 494-515, 2013.

34. Häcker H, Redecke V, Blagoev B, Kratchmarova I, Hsu LC, Wang GG, Kamps MP, Raz E, Wagner H, Häcker G, et al: Specificity in Toll-like receptor signalling through distinct effector functions of TRAF3 and TRAF6. Nature 439: 204-207, 2006.

35. Oganesyan G, Saha SK, Guo B, He JQ, Shahangian A, Zarnegar B, Perry A and Cheng G: Critical role of TRAF3 in the Toll-like receptor-dependent and -independent antiviral response. Nature 439: 208-211, 2006.

36. Mittal RD, Bid HK, Manchanda PK and Kapoor R: Association of interleukin-1beta gene and receptor antagonist polymorphisms with calcium oxalate urolithiasis. J Endourol 21: 1565-1570, 2007.

37. Reyes L, Reinhard M and Brown MB: Different inflammatory responses are associated with Ureaplasma parvum-induced UTI and urolith formation. BMC Infect Dis 9: 9, 2009.

38. Fujii Y, Okada A, Yasui T, Niimi K, Hamamoto S, Hirose M, Kubota Y, Tozawa K, Hayashi Y and Kohri K: Effect of adiponectin on kidney crystal formation in metabolic syndrome model mice via inhibition of inflammation and apoptosis. PLoS One 8: e61343, 2013.

39. Hu X, Wang M, Bei W, Han Z and Guo J: The Chinese herbal medicine FTZ attenuates insulin resistance via IRS1 and PI3K in vitro and in rats with metabolic syndrome. J Transl Med 12: 47, 2014.

40. Kaur J: A comprehensive review on metabolic syndrome. Cardiol Res Pract 2014: 943162, 2014.

41. Sakhaee K, Capolongo G, Maalouf NM, Pasch A, Moe OW, Poindexter J and Adams-Huet B: Metabolic syndrome and the risk of calcium stones. Nephrol Dial Transplant 27: 3201-3209, 2012.

42. Kohjimoto Y, Sasaki Y, Iguchi M, Matsumura N, Inagaki T and Hara I: Association of metabolic syndrome traits and severity of kidney stones: Results from a nationwide survey on urolithiasis in Japan. Am J Kidney Dis 61: 923-929, 2013.

43. Kabeya Y, Kato K, Tomita M, Katsuki T, Oikawa Y, Shimada A and Atsumi Y: Associations of insulin resistance and glycemic control with the risk of kidney stones. Intern Med 51: 699-705, 2012.

44. Khan SR: Is oxidative stress, a link between nephrolithiasis and obesity, hypertension, diabetes, chronic kidney disease metabolic syndrome? Urol Res 40: 95-112, 2012. 